\title{
The open data HELI-DEM DTM for the western alpine area: computation and publication
}

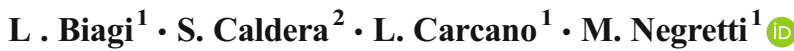

Received: 3 November 2015 / Accepted: 11 October 2016/Published online: 26 October 2016

(C) The Author(s) 2016. This article is published with open access at Springerlink.com

\begin{abstract}
Two problems can arise in analyzing Digital Elevation Models (DEMs). Firstly, one interest region could be patched by several partly overlapping models that present similar accuracies and spatial resolutions; they should be merged in one unified model. Moreover, even when the interest region is completely covered by one model, local data with better accuracy could cover partial areas and should be properly merged. All these problems have been addressed within Helvetia-Italy Digital Elevation Model, a project funded by the European Regional Development Fund (ERDF). One specific aim of the project was the creation and the publication of a unified DEM for a part of the Alps between Italy and Switzerland. The interest area is prevalently mountainous, with elevations that range from about 200 to $4600 \mathrm{~m}$. Various elevation datasets were available that were gridded with different spatial resolutions, in different reference frames and coordinates. Moreover, one high resolution LiDAR model was available for some areas. Firstly, a validation was performed that allowed the identification of few blunders and confirmed the general accuracy of the input data. Two DEMs have been produced. Both of them cover the whole
\end{abstract}

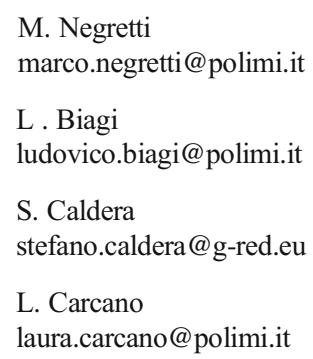

1 DICA at Geomatics Laboratory of Como Campus, Politecnico di Milano, Via Valleggio, 11, 22100 Como, Italy

2 GReD srl, Geomatics Research \& Development, Como, Italy project area (boundaries $\lambda=7.80^{\circ}$ East and $\lambda=10.70^{\circ}$ East, $\varphi=45.10^{\circ}$ North and $\varphi=46.70^{\circ}$ North) and are gridded in ETRF2000 geographical coordinates, with a spatial resolution of $2 \times 10^{-4}$ degrees. The former (named HD-1) has been obtained by interpolating and merging all the low resolution models on a new common grid. HD-1 has been locally corrected by the LiDAR model, where it was available; to avoid sharp discontinuities, the corrections have been filtered by Fast Fourier Transform before applying them. The resulting model has been called HD-2. HD-1 and HD-2 are published by an open access geoservice.

Keyword DTM · Reference frame transformation . Re-gridding $\cdot$ Merging $\cdot$ HELI-DEM

\section{Introduction}

Digital Elevation Models (DEMs, Li et al. 2005) provide a numerical description of the terrain elevation. Stored elevations are typically orthometric heights; when the acquisition technique produces ellipsoidal heights, these are converted into orthometric. DEMs can be classified in Digital Surface Models (DSMs), which represent the actual Earth surface (including buildings, vegetation, etc.) and Digital Terrain Models (DTMs), which represent the elevation of the bare soil.

Different models can be adopted to store and transmit a DEM; grids represent the most popular standard (El-Sheimy et al. 2005). Grids are georeferenced regular matrices of $\left(x_{\mathrm{i}}, y_{\mathrm{i}}\right)$ nodes, whose elevations $H_{\mathrm{i}}$ are stored. The horizontal coordinates of the nodes can be either in a cartographic projection ( $x$ : East, $y$ : North) or geographic $(x: \lambda, y: \varphi)$. Typically, the horizontal spacing between nodes (the grid resolution $\Delta x$ and $\Delta y$ ) are equal in $x$ and $y$ directions; this is not a strict requirement but quite a standard. The georeferencing of a grid requires the 
knowledge of the reference frame, the coordinate system, the grid origin (typically, the coordinates of the upper left node), $\Delta x, \Delta y$, and the total number of rows and columns; additional metadata are needed and are stored in the header. The vertical standard deviation (ZS) of a DEM defines the accuracy of the stored elevations.

The production of global DEMs by remote sensing techniques (Gesch et al. 2006) has grown significantly in the last decade. Their resolutions have improved from about $90 \mathrm{~m}$ of SRTM (v4.1, http://www.cgiar-csi.org/data/srtm-90m-digitalelevation-database-v4-1) to the $12 \mathrm{~m}$ of TerraSAR-X add-on for Digital Elevation Measurement (TanDEM-X). Accuracies reach now the order of few meters; for example, the absolute accuracies of TanDEM-X and ALOS World 3D are respectively of $4 \mathrm{~m}$ (http://www.dlr.de/eo/en/desktopdefault.aspx/ tabid-5727/10086 read-21046/) and $5 \mathrm{~m}$ (http://www.eorc.jaxa. $\mathrm{jp} / \mathrm{ALOS} / \mathrm{en} / \mathrm{aw} 3 \mathrm{~d} 30 /$ ). They are fundamental for global applications and are necessary also at the local scale, where local models are not available. However, local DEMs are preferred where they are available because typically, they are characterized by better accuracies and resolutions. In particular, regional or national models can be produced by aerial photogrammetry with resolution and accuracy of some meters (Li et al. 2005). By LiDAR technique (Brovelli et al. 2004; Liu 2008), high resolution and high accuracy (both better than $1 \mathrm{~m}$ ) models can be produced.

The merging of local bordering models is a major objective especially in alpine areas where hydrogeological analyses are needed (Li and Lihao 2004; Grimaldi et al. 2010); there, one model which covers the whole interest area could be useful to analyze the scenario. However, bordering or overlapping local models cannot be immediately merged, because typically, they are not in the same grid. Moreover, possible inconsistencies must be carefully checked in the border overlaps.

In recent years, Italy and Switzerland have produced different DTMs that have been acquired by different data sources, are characterized by different reference frames, accuracies, and resolutions, and cover different areas of the Alps. HELI-DEM (Helvetia-Italy Digital Elevation Model, Biagi et al. 2011) project, funded by the European Regional Development Fund (ERDF) within the ItalySwitzerland cooperation program, aimed at developing a unique DTM for the alpine and subalpine area between Italy and Switzerland.

HELI-DEM started in 2010 and finished at the end of 2013; the involved institutions were Fondazione Politecnico di Milano, Politecnico di Milano, Politecnico di Torino, Regione Lombardia, Regione Piemonte, and Scuola Universitaria della Svizzera Italiana.

The interest area (Fig. 1) is the alpine parts of the Italian Regions Lombardy, Piedmont and of the Swiss cantons Ticino and Grisons.
To fulfill the project objective, the following intermediate tasks had to be completed:

- collection and analysis of all the available DTMs for the project,

- cross check of them, including cross and external validation,

- integration of the input DTMs to create the final unified DTM,

- and free and open source distribution of the final unified DTM.

In the following sections, all the performed tasks are described. In particular, the "Data preprocessing" section summarizes the data preprocessing, the "Computation of HELI-DEM1 " section discusses the merging of low resolution DTMs, the "Computation of HELI-DEM-2" section discusses the corrections with a high resolution LiDAR DTM, and the "HD-1 and HD-2 publication" section presents the implementation of a geoservice for the publication of the final results.

\section{Data preprocessing}

Preprocessing includes all the needed operations to prepare the input data. Firstly, the available data are collected and then they are checked. Our available data can be mainly classified in the following ways (Biagi et al. 2012):

1. Regional low resolution (LR) models. They are the three DTMs officially released by Lombardy (LO-DTM), Piedmont (PI-DTM), and Switzerland (DTMCH-25). LO-DTM is gridded in Roma40-GB coordinates, with an horizontal resolution of $25 \mathrm{~m}$ and nominal $\mathrm{ZS}$ of about $10 \mathrm{~m}$ in mountains. PI-DTM is in ETRF89-UTM coordinates, with resolution of $50 \mathrm{~m}$ and nominal ZS of $5 \mathrm{~m}$. DTMCH-25 is in ETRF89 geographic coordinates, with resolution of 1" (about $31 \mathrm{~m}$ in $\varphi$ and $22 \mathrm{~m}$ in $\lambda$ ) and nominal ZS of about $2-3 \mathrm{~m}$.

2. One high resolution (HR) LiDAR DTM. It is named PSTA, has been realized by the Italian Ministry of Environment, and covers the main river basins of Lombardy and Piedmont. It is in ETRF89 geographic coordinates, with a resolution of $0.00001^{\circ}$ (about $1 \mathrm{~m}$ ) and nominal ZS of $10 \mathrm{~cm}$.

Input data are in different reference frames. The output model should be in ETRF2000, i.e., the present official European Reference Frame. Therefore, to harmonize the data, transformations of reference frames are needed; for this purpose, specific libraries (Biagi et al. 2012) have been developed that implement international EUREF and Italian specifications (Altamimi and Boucher 2001; Donatelli 
Fig. 1 Area of interest of HELIDEM project

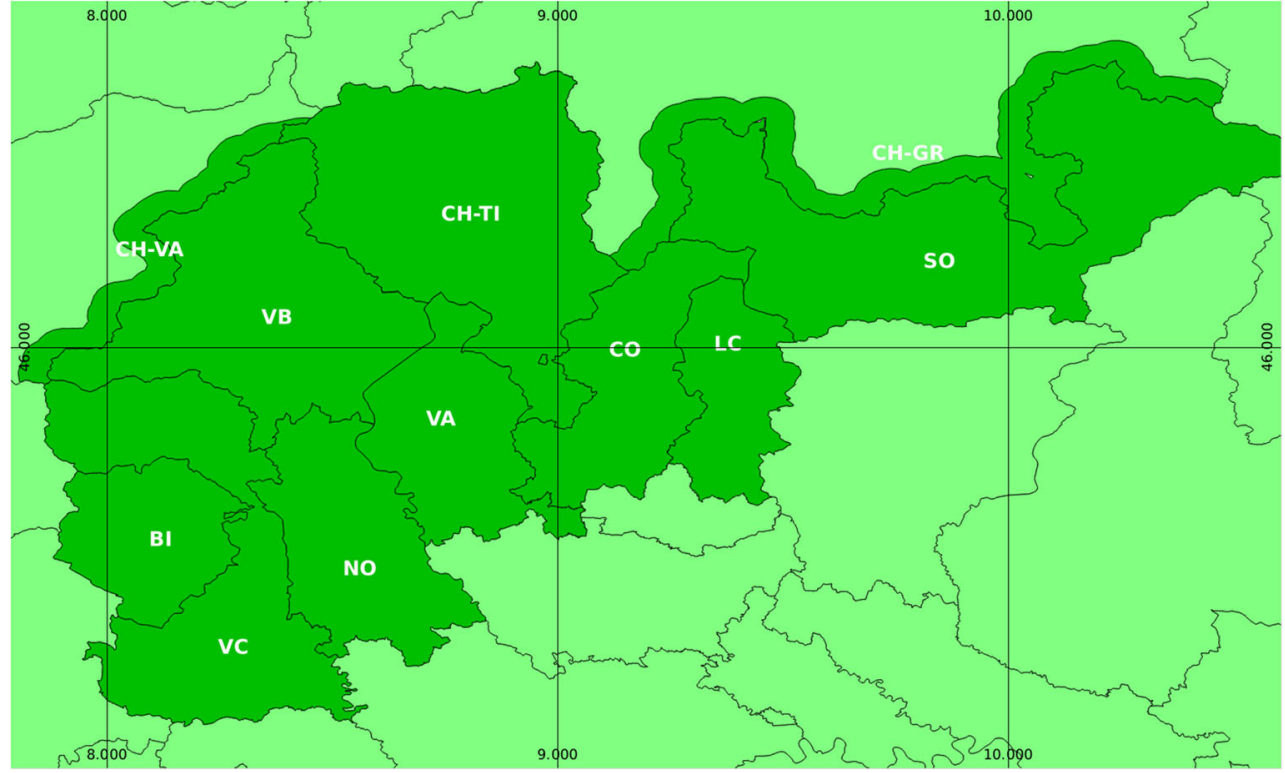

Checks of low resolution models with PST-A have generally provided satisfactory results; this was in some way expected, because PST-A covers only the low valleys where less problems are expected than on slopes and mountains. Only in Val Pola (Valtellina area), anomalous localized values exist with a maximum difference of about $100 \mathrm{~m}$ (Fig. 3). This is due to a huge landslide that occurred in 1987 (Costa 1991); LO-DTM was produced before the event, while PST-A is more recent.

Ad hoc real-time kinematic (RTK) surveys in the anomalous areas confirmed the correctness of PST-A (Biagi et al. 2013a), whose use to correct less accurate values should generally be planned.

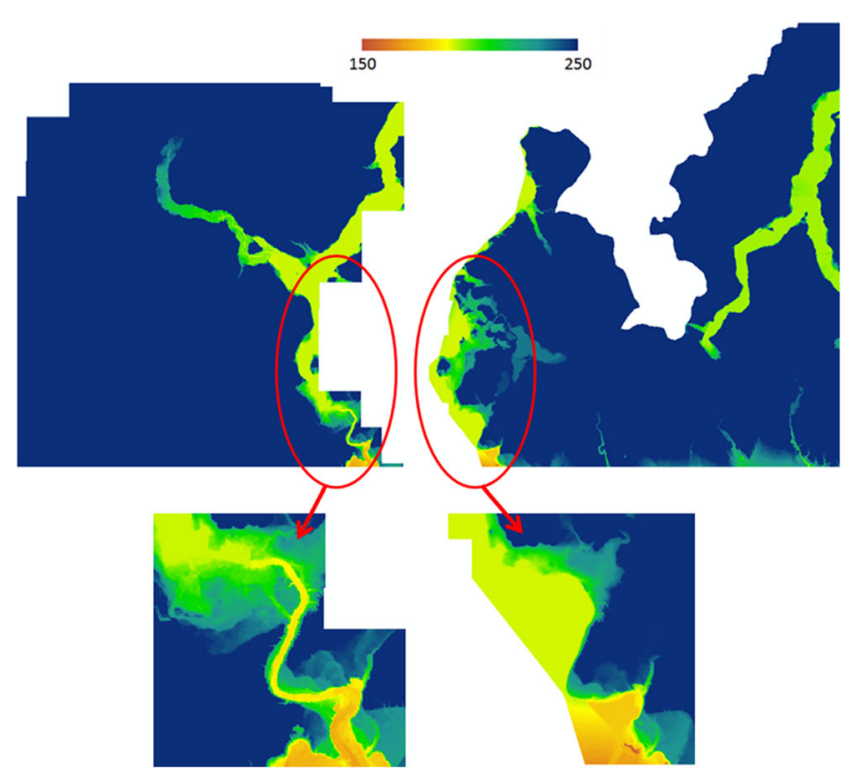

Fig. 2 PI-DTM (on the left) and LO-DTM (on the right) in Lago Maggiore and Ticino valley. The red circle highlights the inconsistent data 
Fig. 3 LO-DTM (left) and its differences with PST-A (right) in the Val di Pola landslide area

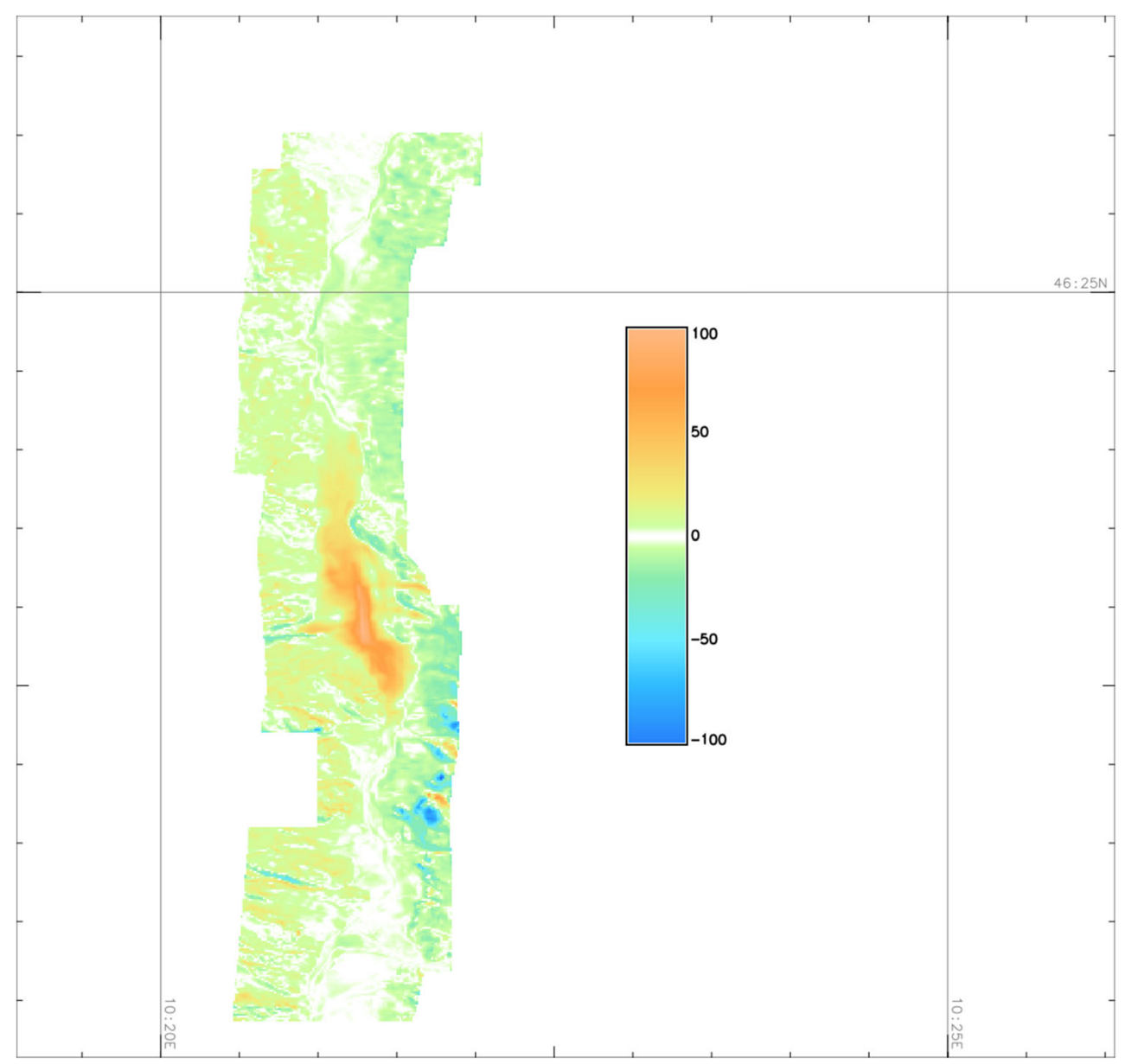

\section{Computation of HELI-DEM-1}

One DTM which covers the whole interest area must be created. In particular, the output DTM must be in ETRF2000 geographic coordinates and must cover the rectangle inside the following boundaries: $\lambda=7.80^{\circ} \mathrm{E}, \lambda=10.70^{\circ} \mathrm{E}, \varphi=45.10^{\circ} \mathrm{N}$ and $\varphi=$ $46.70^{\circ} \mathrm{N}$. The adopted spatial resolution is $2 \cdot 10^{-4}$ sexagesimal degrees (about $15 \mathrm{~m}$ in longitude and $22 \mathrm{~m}$ in latitude).

All the input DTMs must be transformed to ETRF2000. Note that a reference frame transformation of a point is a three dimensional transformation of its $X, Y, Z$ (or $\varphi, \lambda, \mathrm{h}$ ) coordinates. However, a point of a DTM is a node of an horizontal grid whose orthometric height is stored; therefore, the reference frame transformation is applied only to the horizontal coordinates of the node. The output is a list of transformed horizontal points and their orthometric heights; the points are again almost regularly distributed but no more on an exactly oriented grid. Moreover, a re-gridding could be needed with a different resolution or in a different coordinate system. Different procedures can be adopted to transform, re-grid, and merge more DTMs; in general, three independent operations are necessary regardless of the order:
- transformation of the input DTMs to a common reference frame,

- re-gridding of the DTMs on the output grid,

- and merging of the re-gridded data.

In order to perform them, at least two different approaches can be applied.

1. Firstly, the input DTMs are transformed to the output reference frame; the resulting datasets are individually re-gridded on the output grid. Finally, the obtained grids are merged by averaging in the overlapping areas. This approach is called direct.

2. The horizontal coordinates of the output grid nodes are back transformed from the output reference frame to those of the input models. Each input DTM, still gridded in its own reference frame, is interpolated on these horizontal points. The interpolated elevations are then assigned to the output nodes. Finally, the obtained grids are merged by averaging in the overlapping areas. This approach is called inverse. 


\section{Re-gridding choices}

To re-grid, firstly, a parametric model is chosen; the parameters are then estimated by the input data. Many approaches and models exist; a first classification can be in interpolation and approximation (Christakos 1992). In interpolation, a model is estimated that passes through all the observations. In approximation, statistical methods are applied to estimate a smoother model from the observations.

When raw observations are used to estimate a model, approximation must be adopted because this allows the filtering of errors and outliers; one example is discussed in Biagi and Negretti (2004). On the contrary, in our case, the input data came from models that have been already filtered and checked against errors. In this case, actual details can be lost by smoothing while interpolation should provide better results.

Typically, geographic information system (GIS) software (O’Sullivan and Unwin 2003) implements either splines or polynomial surfaces (Kidner 2003) to interpolate grid nodes. Particularly, we have focused on the use of bilinear and bicubic surfaces; previous works (see for example Rees 2000) stated the better accuracy of bicubic surfaces and our preliminary analyses confirmed that. A bicubic surface is given by the following:

$$
\begin{aligned}
H(x, y) & =a_{00}+a_{10} x+a_{01} y+a_{20} x^{2}+a_{11} x y+a_{02} y^{2} \\
& +a_{30} x^{3}+a_{21} x^{2} y+a_{12} x y^{2}+a_{03} y^{3} \\
& +a_{31} x^{3} y+a_{22} x^{2} y^{2}+a_{13} x y^{3}+a_{32} x^{3} y^{2}+a_{23} x^{2} y^{3}+a_{33} x^{3} y^{3}
\end{aligned}
$$

Firstly, let us consider the generic case of sparse observations and their interpolation or approximation in a point $P=\left(x_{P}, y_{P}\right)$. At least $16\left(x_{i}, y_{i}, H_{i}\right)$ observations around $P$ are needed to estimate $a_{i j}$ parameters; the system can be written as follows:

$\mathbf{z}=\left[\begin{array}{c}H_{1} \\ H_{2} \\ \ldots \\ H_{n}\end{array}\right]=\mathbf{A} \xi=\left[\begin{array}{ccccc}1 & x_{1} & y_{1} & \ldots & x_{1}^{3} y_{1}^{3} \\ 1 & x_{2} & y_{2} & \ldots & x_{2}^{3} y_{2}^{3} \\ \ldots & \ldots & \ldots & \ldots & \ldots \\ 1 & x_{n} & y_{n} & \ldots & x_{n}^{3} y_{n}^{3}\end{array}\right]\left[\begin{array}{c}a_{00} \\ a_{10} \\ a_{01} \\ \ldots \\ a_{33}\end{array}\right]$

If exactly 16 observations are used, the parameters are estimated by the iso-determined system.
$\xi=\mathbf{A}^{-1} \mathbf{z}$

Clearly, the solution requires that $\mathbf{A}$ matrix is well conditioned. Finally, the estimated parameters are used to estimate elevations in $P$. In this case, the bicubic surface acts as interpolator.

If more than 16 observations are used, a redundant system is built and can be solved by the least squares (LS, Koch 1987) approach.

$\xi=\mathbf{N}^{-1} \mathbf{A}^{T} \mathbf{Q}^{-1} \mathbf{z}, \mathbf{N}=\mathbf{A}^{T} \mathbf{Q}^{-1} \mathbf{A}$

Also in this case, the good conditioning of the normal system is required; again, the estimated parameters are used to estimate elevations in $P$. In this case, the bicubic surface acts as approximation.

In the case of direct approach, input points must be managed as sparse observations and Eqs. (3) or (4) must be implemented; methodological and technical problems of this approach are extensively discussed in Biagi and Carcano (2015). In the paper, a particular attention is given to the numerical regularization of ill-conditioned cases that can occur in specific geometric configurations, even if the input data are regularly distributed.

On the opposite, let us now consider the bicubic interpolation of gridded data; in this case, to interpolate in a point $P$, the 16 nodes of the 4 rows $\times 4$ columns around it can be used. The system Eq. (2) is solved by five consecutive cubic interpolations along lines, without any matrix inversion, as is shown in Press et al. (2007) and applied in Carcano (2014). Let us suppose that $P$ is between rows $i$ and $i+1$ and columns $j$ and $j+1$ of the matrix DTM; let us call $I$ and $J$ the row and column real valued coordinates of $P$. In the first step, the four points in each row, $k, k=i-1, i, i+1, i+2$, are used to interpolate a point in $k, J$ :

$$
\begin{array}{lll}
H_{i-1, j-1}, H_{i-1, j}, H_{i-1, j+1}, H_{i-1, j+2} & \rightarrow & H_{i-1, J} \\
H_{i, j-1}, H_{i, j}, \quad H_{i, j+1}, \quad H_{i, j+2} & \rightarrow & H_{i, J} \\
H_{i+1, j-1}, H_{i+1, j}, H_{i+1, j+1}, H_{i+1, j+2} & \rightarrow & H_{i+1, J} \\
H_{i+2, j-1}, H_{i+2, j}, H_{i+2, j+1}, H_{i+2, j+2} & \rightarrow & H_{i+2, J}
\end{array}
$$

More explicitly,

$$
\begin{aligned}
H_{k, J} & =-\frac{1}{6}(J-j)(j+1-J)(j+2-J) H_{k, j-1}+\frac{1}{2}(J+1-j)(j+1-J)(j+2-J) H_{k, j}+ \\
& +\frac{1}{2}(J+1-j)(J-j)(j+2-J) H_{k, j+1}-\frac{1}{6}(J+1-j)(J-j)(j+1-J) H_{k, j+2}
\end{aligned}
$$

In the second step, $H_{i-1, J}, H_{i, J}, H_{i+1, J}$, and $H_{i+2, J}$ are interpolated in $I$ :

$$
\begin{aligned}
H_{I, J} & =-\frac{1}{6}(I-i)(i+1-I)(i+2-I) H_{i-1, J}+\frac{1}{2}(I+1-i)(i+1-I)(i+2-I) H_{i, J}+ \\
& +\frac{1}{2}(I+1-i)(I-i)(i+2-I) H_{i+1, J}-\frac{1}{6}(I+1-i)(I-i)(i+1-I) H_{i+2, j+2}
\end{aligned}
$$


The same approach applied in the opposite order produces the same results that are exactly equal to the solution of Eq. (2) for this particular spatial configuration. No system inversion is needed and the solution is always well conditioned. This twostep process is simply an application of the Lagrange polynomial interpolator in the special case of equidistant data.

This is exactly the interpolation needed in the inverse regridding, where input data are still gridded. In case less than 16 elevations exist around $P$, the interpolation cannot be computed and the result is put to "no data"; this happens at the borders of the input grid (and clearly outside it).

\section{Final interpolation of HD-1}

Firstly, direct approach was implemented to compute HD-1; the applied procedure is described in Biagi et al. (2013b). Unfortunately, when extensively applied to the whole input dataset, the direct approach provided numerical problems in a significant number of points.

Therefore, computation strategy has changed and the inverse approach has been implemented and tested. The ETRF2000 horizontal coordinates of the output nodes are transformed to the reference frame of the three input DTMs that are interpolated by Eq. (5a) and (5b) on these points. The interpolated elevations are assigned to the output nodes. To cross check, the process can be reversed; interpolated grids are back interpolated on the input nodes. Original and twiceinterpolated elevations are compared. Table 1 reports the cross checks for LO-DTM, PI-DTM, and DTMCH-25. In case of PIDTM and DTMCH-25, results are completely satisfactory; the differences are not significant with respect to the nominal accuracies of input data. LO-DTM results are satisfactory but worse than others; the worst differences are in very rough areas.

At the end, three interpolated DTMs are available and must be merged; they share the same grid (origin, spatial resolution, and number of nodes) but each one contains elevations only inside the domain of its input DTM, while the external nodes contain "no data".

Most of the nodes of the final grid contain just one elevation that is the final stored elevation. On the contrary,

Table 1 Cross comparisons between input and back-interpolated elevations for LO-DTM, PI-DTM, and CHM25

\begin{tabular}{llll}
\hline & LO-DTM & PI-DTM & DTMCH-25 \\
\hline$N\left(10^{+6}\right)$ & $\approx 30 \mathrm{M}$ & $\approx 3.4 \mathrm{M}$ & $\approx 12 \mathrm{M}$ \\
Mean (m) & 0.0 & 0.0 & 0.0 \\
Std. dev. (m) & 0.8 & 0.2 & 0.3 \\
Min. (m) & -52 & -6.8 & -17.8 \\
Max. (m) & 48 & 6.7 & 17.9
\end{tabular}

$N$ number of points (in million), Mean mean difference, Std. dev. standard deviation, Min. minimum difference, Max. maximum difference interpolated DTMs overlap at their borders. To merge them, existing discontinuities must be smoothed; a weighted approach has been applied. Let $P$ be a node where more DTMs overlap; the horizontal distances of $P$ from the borders of all the overlapping DTM can be computed by standard GIS functions (Fig. 4). The final elevation in $P$ is computed by the following:

$h=\frac{1}{\sum_{i} d_{i}} \sum_{i} d_{i} h_{i}$

where $h_{i}$ are the elevations of overlapping DTMs in $P, d_{i}$ are the distances between $P$ and the borders of overlapping DTMs. Note that this weighting has the purpose to avoid discontinuities at the borders of overlaps and not statistical reasons.

The final DTM (HD-1) is shown in Fig. 5 and its statistics are summarized in Table 2.

\section{Computation of HELI-DEM-2}

The high resolution PST-A DTM is available in the river basins areas and can be used to improve HD-1. The simple replacement of HD-1 values with PST-A can introduce local significant discontinuities and a proper filtering has to be applied. The proposed procedure can be schematized in the following ways:

1. computation of the corrections,

2. filtering of the corrections,

3. and application of the filtered corrections to HD-1.

Computation of the corrections PST-A is subsampled on HD-1 nodes: firstly, all the nodes of PST-A within the square domain of each HD-1 node are averaged and then the average is assigned to the HD-1 node. The matrix containing the

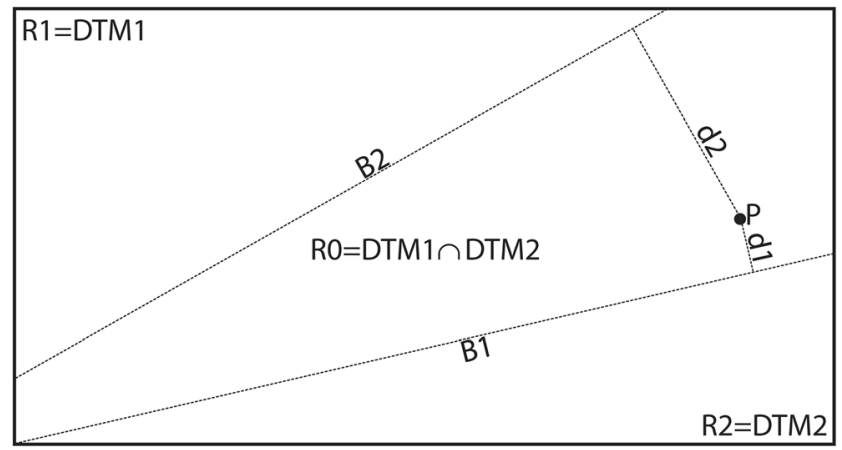

Fig. 4 Weighted average in the overlaps. $R 1$ : domain of DTM1. $R 2$ : domain of DTM2. R0: overlap of DTM1 and DTM2. $B 1$ : border of DTM1. B2: border of DTM2. $d 1$ and $d 2$ : distances of $P$ from $B 1$ and $B 2$. 
Fig. 5 HD-1 DTM. Elevations in meters.

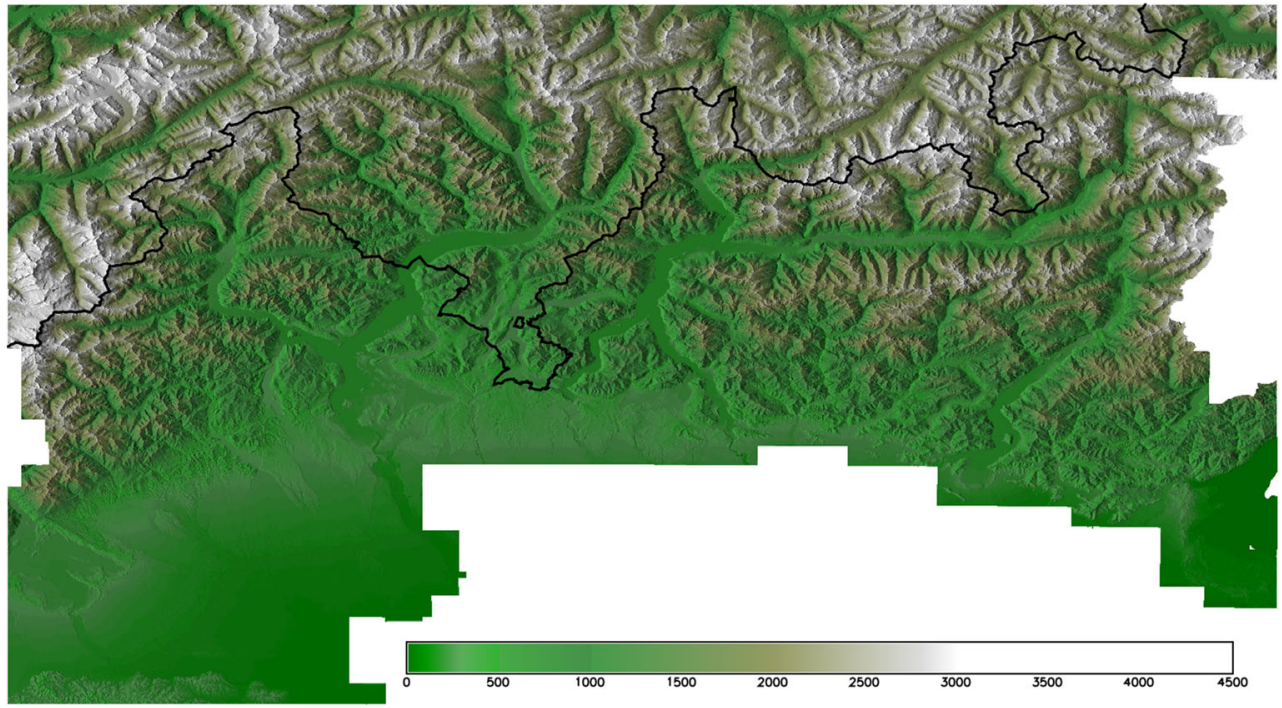

differences between PST-A and HD-1 is computed: the differences corresponding to PST-A "no data" are set to zero. This is the correction model.

Filtering of the corrections by low pass filter Due to the subsampling of PST-A, the correction model is already smoothed; however, discontinuities at the borders can be in principle sharp. A low pass filter allows to smooth the corrections at their borders; it can be described as the convolution of a signal with a mask whose integral is equal to one. The low pass Butterworth filter is defined as follows:

$f_{n}(d)=\frac{1}{1+d^{2 n} / D_{0}^{2 n}}$

where $n$ is the order of the filter, $D_{0}$ is the halving distance. In the bi-dimensional discrete case, the mask is a matrix of coefficients that are null outside a given window.

$f_{n}(i, j)=\frac{k}{1+\left(i^{2}+j^{2}\right)^{n} / D_{0}^{2 n}}$

Table 2 Statistics of the elevations of HD-1 DTM

\begin{tabular}{ll}
\hline Statistic of final grid & \\
\hline Number of grid nodes (millions) & $116 \mathrm{M}$ \\
Number of stored elevations (millions) & $88.5 \mathrm{M}$ \\
Mean & $1301 \mathrm{~m}$ \\
Std. dev. & $920 \mathrm{~m}$ \\
Max. & $4616 \mathrm{~m}$ \\
Min. & $17 \mathrm{~m}$
\end{tabular}

Mean mean elevation, Std. dev. standard deviation, Min. minimum elevation, Max. maximum elevation
Here, indexes $i, j$ are the node positions with respect to the origin of the filter (convolution application). In our application, we chose $D_{0}=5$ and $n=2$. The dimension of the square filter was $11 \times 11$; $k$ is a constant to normalize the sum of its coefficients.

The filtering has been implemented by a Fast Fourier Transform (FFT) approach (Brigham 1974):

1. construction of the Butterworth filter matrix.

2. computation of FFTs of both correction model and Butterworth filter,

3. product of the two FFTs,

4. and inverse FFT of the product.

To visually analyze the filtering benefits, just the results of a small case study (Oglio river) are here reported. Figure 6 shows the original and the filtered corrections; the filtering effect is evident at the borders.

The filtered corrections have been applied to the unified DTM. The output DTM is called HD-2. Internal and external validations are useful to evaluate if the correction actually leads to an improvement of the final product. As internal validation, HD-1 and HD-2 are compared to PST-A DTM. The statistics of the differences demonstrate the usefulness of the correction. In Oglio river valley, the mean and the standard deviation equal respectively to -1.9 and $5.8 \mathrm{~m}$ before the correction and 0.0 and $1.1 \mathrm{~m}$ after the correction. In the complete HD-1, they are equal to 0.3 and $6.0 \mathrm{~m}$ before the correction and 0.0 and $0.4 \mathrm{~m}$ after.

As external validation, HD-1 and HD-2 are compared with about 1000 checkpoints that are available in some test areas. They have been surveyed by GNSS RTK, with an accuracy surely better than $10 \mathrm{~cm}$ and have been used in a previous step of the project to check LO-DTM where it has provided anomalous values (Biagi et al. 2013a); in particular, they are located 

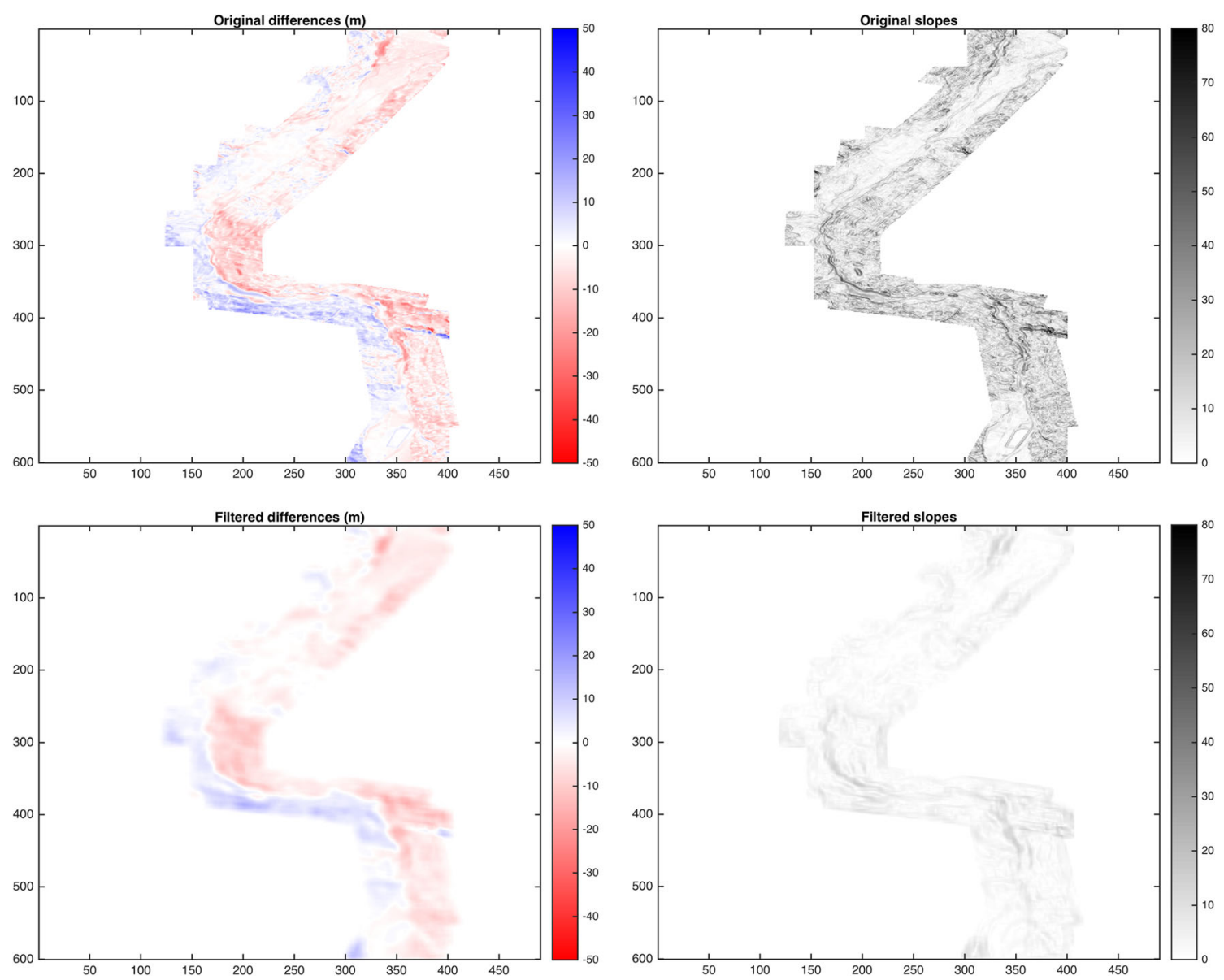

Fig. 6 The filtering effect shown for a small case study (Oglio river, $600 \times 500$ cells). Above: original correction model (differences between subsample PST-A and HD-1). Left: differences. Right: slopes. Below: filtered correction model. Left: differences. Right: slopes.

in 12 test areas of Valtellina for a total number of 943 test points. To compare, GNSS geodetic heights have been converted to orthometric heights by applying ItalGeo2005 (Barzaghi et al. 2007). The subsampled PST-A and HD-2 have been interpolated by bicubic interpolation on the test points, and the differences with respect to test values have been computed (Table 3). The statistics prove the reliability of the applied correction; indeed, the results of HD-2 are significantly better than those of HD-1 and are comparable with those of PST-A.

As example, Fig. 7 shows on the same graphs the differences between HD-1, PST-A (in this case not subsampled),

Table 3 Statistics of the differences between HD-1, PST-A, and HD-2 DTMs and RTK

\begin{tabular}{lllc}
\hline & Mean $(\mathrm{m})$ & Std dev $(\mathrm{m})$ & Max $(\mathrm{m})$ \\
\hline HD-1-RTK & 3.4 & 5.5 & 24.2 \\
PST-A-RTK & -0.3 & 1.0 & 7.2 \\
HD-2_RTK & -0.4 & 1.7 & 8.8 \\
\hline
\end{tabular}

Mean mean difference, Std dev standard deviation, Max maximum difference
HD-2, and the 64 points of one area that were surveyed along an almost straight path. A bias of HD-1 is clear, with some points that present differences up to $11 \mathrm{~m}$. The differences between PST-A and RTK are hardly recognizable at the scale of representation. HD-2 differences are more similar to the high resolution case.

\section{HD-1 and HD-2 publication}

In publishing HD-1 and HD-2 data, our main goal was to ensure the data access in a simple way. To realize a high level of data accessibility and make easy to use them, we decided to implement standards; Open Geospatial Consortium (OGChttp://www.opengeospatial.org/) provides the main benchmarks. OGC is an international consortium of more than 500 members: companies, government agencies and universities participating to the development and use of international standards and supporting services that promote geospatial interoperability.

For our application, the OGC web service (OWS) standards must be adopted to implement the GeoWeb services that 
Fig. 7 Differences $(\mathrm{cm})$ between HD-1/HD-2/PST-A and RTK points in area VT02 in Valtellina Valley

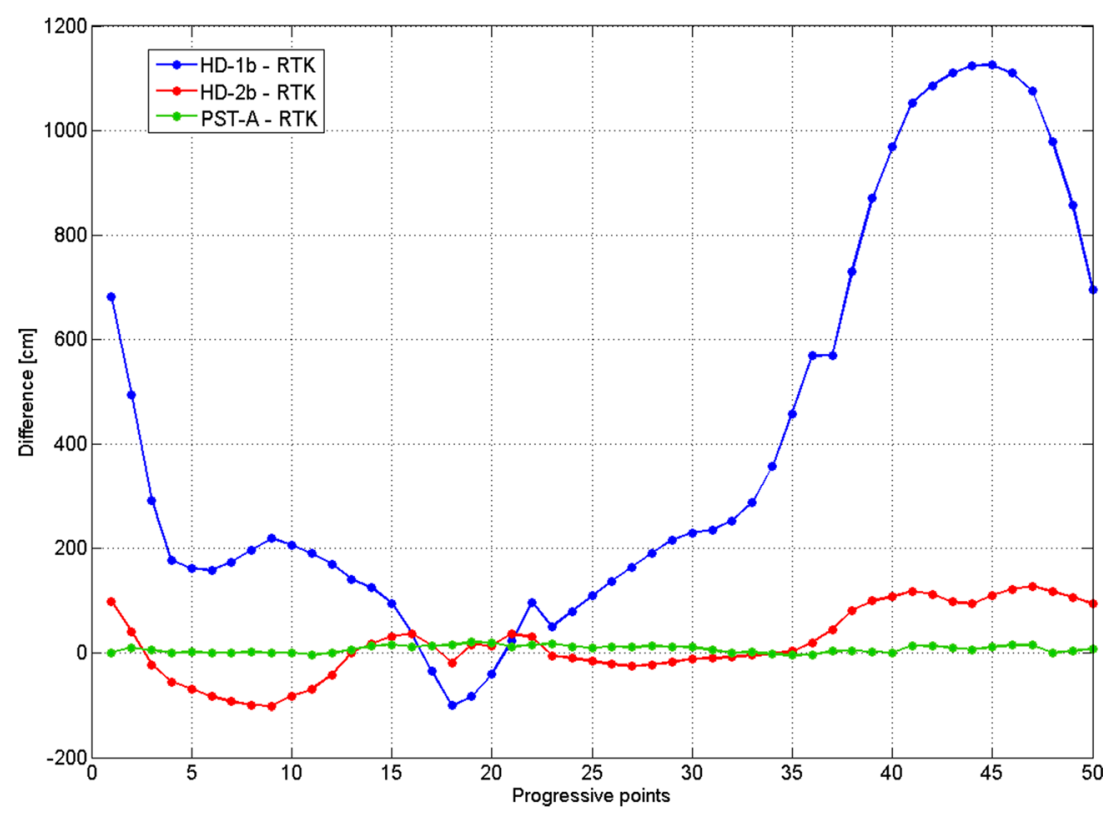

are needed to distribute spatial data (Peng and Tsou 2003 and Brovelli et al. 2011).

Using OWS, data and geoservices can be published to anyone that is standard compliant, independently from an operative system, GIS software, or other client properties or configuration (Michaelis and Ames 2007).

Figure 8 shows the basic scheme of an OWS service. The client sends a standard request to the server. Then, the server accesses to the data, processes them according to the request, and finally sends the response to the client. Many different requests can be managed, depending on the type of OWS service implemented. Basically, they can be grouped in four main kinds:

- "Get capabilities" requests to which the server responds with a list the process or data available,

- "Describe" requests to which the server responds with the description of a particular datum or process, and

- "Get data" or "Get service" requests to which the server responds delivering data or executing specific processes.

Moreover, whether the server implements transaction functionalities, also data editing requests exist.

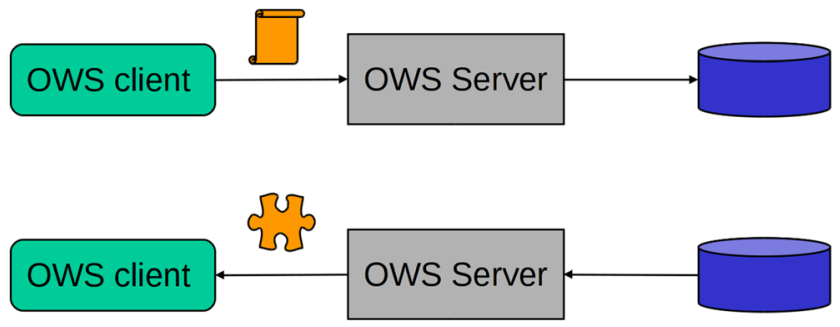

Fig. 8 Basic scheme of an OWS service
Different standards are defined, according to the type of the data published in the geoserver; our data are grids (geo referenced raster data). Web coverage service (WCS) (Baumann 2012) and Web map service (WMS) (De La Beaujardiere et al. 2006) have been implemented.

WCS (http://www.opengeospatial.org/standards/wcs) publishes raster data as georeferenced numeric matrices; the user can use them for processing and any other needed activity. Data published by WCS can be heavy; if you need them only for background images, the full numeric matrix is actually useless.

WMS (http://www.opengeospatial.org/standards/wms) publishes data as simple georeferenced image. Data published by WMS are lighter; therefore, we have implemented also this service.

To implement WCS and WMS, we adopted GeoServer (http://geoserver.org/). GeoServer is a Java-based software that allows to access and edit geospatial data; it is free and open source and uses the open standards set by the OGC Our geoservice with HD-1 and HD-2 is available at the following address of Geomatics Laboratory of Como Campus of Politecnico di Milano (http://geomatica.como.polimi.it; http://www.helidemdataserver.como.polimi.it:8080/ geoserver/ows).

\section{Conclusions}

The HELI-DEM project was aimed at developing a unique DTM which covers the alpine area between Lombardy, Piedmont, Ticino, and Grisons Cantons. For this project, different DTMs were available, acquired with different accuracies and resolutions and in different reference frames: at low/ 
medium resolution and accuracy, the two official DTMs of Italian Regions Lombardy and Piedmont and the national Swiss DTM and at high resolution, a LiDAR DTM which covers the main Italian river basins. These datasets have been merged to create one model.

To do that, several preliminary analyses have been performed, with the purpose to cross check and to assess the accuracy of all the input datasets. The performed analyses confirmed the general quality of the available data and allowed to identify and eliminate few anomalies.

Output model HD-1 has been obtained by re-gridding and merging of the low resolution available DTMs. Cross checks of HD-1 with input datasets confirm the correctness of adopted methods. In area where geodetic benchmarks are available and ad hoc RTK GNSS surveys were made (mainly in Valtellina Valley), the vertical accuracy of the model can be externally checked; it is of the order of $5 \mathrm{~m}$.

Moreover, a method to correct a low resolution model by using high resolution data without introducing discontinuities has been studied and implemented. This method has been applied to HD-1 by using the LiDar model PST-A that was produced by the Italian Ministry of Environment for Italian alpine valleys. The correction, implemented through a Fast Fourier Transform, proved to be effective and the final product is called HD-2. Where corrections existed and have been applied, the final accuracy of HD-2 increases to about $1.7 \mathrm{~m}$.

Both HD-1 and HD-2 are freely published by a FOSS geoservice.

Acknowledgments The present work has been supported by the HELIDEM project, within the Italy-Switzerland cooperation program.

Open Access This article is distributed under the terms of the Creative Commons Attribution 4.0 International License (http:// creativecommons.org/licenses/by/4.0/), which permits unrestricted use, distribution, and reproduction in any medium, provided you give appropriate credit to the original author(s) and the source, provide a link to the Creative Commons license, and indicate if changes were made.

\section{References}

Altamimi Z Boucher C (2001) The ITRS and ETRS89 relationship: new results from ITRF2000, report on the symposium of the IAG subcommission for Europe (EUREF), Dubrovnik

Barzaghi R, Borghi A, Carrion D, Sona G (2007) Refining the estimate of the Italian quasi-geoid. Bollettino di Geodesia e Scienze Affini, ISSN 0006-6710, fascicolo 3/2007, pp. 145-159

Baumann P (2012) OGC WCS 2.0 Interface standard-core, version 2.0.1, OGC Document Number: 09-110r4

Biagi L, Carcano L (2015) Regridding and merging overlapping DTMs: methodological problems and solutions in HELI-DEM. Proceedings of VIII Hotine Marussi symposium on mathematical geodesy, IAG symposia volumes series

Biagi L, Negretti M (2004) Environmental thematic maps prediction and easy probabilistic classification. Trans GIS Vol. 8(Iss. 2)
Biagi L, Brovelli MA, Campi A, Cannata M, Carcano L, Credali M, De Agostino, M, Manzino A, Sansò F, Siletto G (2011) Il progetto HELI-DEM (Helvetia-Italy Digital Elevation Model): scopi e stato di attuazione, in Bollettino della Società Italiana di Fotogrammetria e Topografia, $\mathrm{n}^{\circ} 1 / 2011$, pp. $35-51$, ISSN: $1721-971 \mathrm{X}$

Biagi L, Carcano L and De Agostino M (2012) DTM cross validation and merging: problems and solutions for a case study within the HELIDEM project. Int. Arch. Photogramm. Remote Sens Spatial Inf Sci XXXIX-B4, 2012

Biagi L, Carcano L, Lucchese A, Negretti M (2013a) Creation of a multiresolution and multiaccuracy DTM: problems and solutions for HELI-DEM case study. Int. Arch. Photogramm. Remote Sens. XL-5/W3, The Role of Geomatics in Hydrogeological Risk. Padua

Biagi L, Manzino AM, Sansò F Eds. (2013b) HELI-DEM: integrazione dei dati di altezza transalpini fra Italia e Svizzera, Numero speciale SIFET $5 / 2013$

Brigham EO (1974) The Fast Fourier Transform. by Prentice-Hall, Inc. Englewood Cliffs, N. J.

Brovelli MA, Cannata M, Longoni U (2004) LIDAR data filtering and DTM interpolation within GRASS. Trans GIS 8(2):155-174

Brovelli MA, Giori G, Mussin M, Negretti M (2011) Improving the monitor of the status of the environment through web geo-services: the example of large structures supervision. TGIS 15(Issue 2):173-188

Carcano L (2014) Merging local DTMS: HELI-DEM project, problems and solutions. PhD Thesis. https://www.politesi.polimi. it/bitstream/10589/89463/1/2014 $03 \mathrm{PhD}$ Carcano.pdf

Christakos G (1992) Random field models for earth sciences. Academic Press, New York

Costa JE (1991) Nature, mechanics, and mitigation of the Val Pola landslide, Valtellina, Italy, 1987-1988. Zeitschrift fur Geomorphologie 35

De La Beaujardiere J, Open GIS (2006) Web map server implementation specification, version 1.3.0, OGC document number: 06-042

Donatelli D, Maseroli R, Pierozzi M (2002) La trasformazione tra sistemi di riferimento utilizzati in Italia, Bollettino di Geodesia e Scienze Affini, Anno LXI, $\mathrm{N}^{\circ} 2$

El-Sheimy N, Valeo C, Habib A (2005) Digital terrain modeling-acquisition, manipulation and applications. Artech House

Gesch DB, Muller JP, Farr TG (2006) The shuttle radar topography mission - data validation and applications. Photogramm Eng Remote Sens $72(3)$

Grimaldi S, Petroselli A, Alonso G, Nardi F (2010) Flow time estimation with variable hillslope velocity in ungauged basins. Adv Water Resour 33(10):216-1223

Kidner DB (2003) Higher-order interpolation of regular grid digital elevation models. Int J Remote Sens 24:2981-2987

Koch KR (1987) Parameter estimation and hypothesis testing in linear models, Springer Verlag

Li J, Lihao Z (2004) Distributed hydrologic model based on dem. Hydroelectric Energy 4:002

Li Z, Zhu Q, Gold C (2005) Digital terrain modeling: principles and methodology. CRC

Liu X (2008) Airborne LiDAR for DEM generation: some critical issues. Prog Phys Geogr 32(1)

Michaelis CD, Ames D P (2007) Evaluation and implementation of the OGC web processing service for use in client-side GIS. OSGeo Journal 1:50-55

O'Sullivan D, Unwin D (2003) Geographic information analysis. John Wiley \& Sons., Inc., Hoboken

Peng ZR, Tsou MH (2003) Internet GIS: distributed geographic information services for the Internet and wireless network. John Wiley and Sons, New York

Press WH, Teukolsky SA, Vetterling WT, Brian PF (2007) Numerical recipes in $\mathrm{C}$ : the art of scientific computing, third edition, Cambridge University Press

Rees WG (2000) The accuracy of digital elevation models interpolated to higher resolutions. Int J Remote Sens 21(1):7-20 